\title{
SIMULATION OF THE NUCLEAR FUEL ASSEMBLY DROP TEST WITH LS-DYNA
}

\author{
P.Petkevich $^{1}$, V.Abramov ${ }^{1}$, V.Yuremenko ${ }^{1}$, V.Piminov ${ }^{1}$, V.Makarov ${ }^{1}$, A.Afanasiev ${ }^{1}$ \\ ${ }^{1}$ Experimental and Design Organization "GIDROPRESS", Podolsk, RUSSIA \\ E-mail of corresponding author: petya2306@gmail.com
}

\begin{abstract}
Transportation of the nuclear fuel containing objects is especially sensitive to accidental drops, as any event, affecting the fuel spacial arrangement, alters also neutron multiplication factor and can result in uncontrolled chain reaction. The latter is particularly important for nuclear fuel being immersed in water. Apart from that, fall can result in a mechanical damage of the fuel rods, which can cause environmental pollution by radionuclides.

Final and intermediate fuel configurations during the accident depend on the impact velocity and the angle between falling object and the surface. Experiments cannot cover all the possible variants of drops, as it would result in their unacceptable prices. Therefore elaboration of the approaches to numerically simulate such kind of accidents is an essential step in the nuclear fuel transportation safety analysis and is the principal goal of the present research.

Series of drop tests with fuel assemblies (FA) models of different complexity have been performed and numerically simulated with LS-Dyna software in order to proof the reliability of such kind of analysis. The paper contains description of the drop test experimental facility, some experimental results and their numerical simulation. It has been found that the finite element model of the FA and the material properties used for the simulation provide reliable predictions of the FA materials deformation and failure in case of accidental drops onto a rigid surface.
\end{abstract}

\section{INTRODUCTION}

Transporting of nuclear fuel is potentially dangerous as any accident, affecting geometry or arrangement of the nuclear fuel, affects also neutron multiplication factor. Therefore nuclear safety during transportation has to be provided in any situation which is a Russian nuclear authorities requirement. To analyze neutronics of damaged nuclear fuel one has to be able to properly predict fuel deformations during and after the accident. LS-Dyna software $[1,2]$ is being currently used at EDO "Gidropress" for these purposes. However reliable analysis is hardly possible without thorough verification of the code for certain analysis types. Series of drop tests have been performed in order to proof the reliability of FA accidental drops numerical simulation. Numerical simulation of the experiments has shown a very good agreement between the results, which means that deformations of FA in accidental situations can be analyzed without expensive and complicated experiments.

\section{PECULIARITIES OF THE FA NUMERICAL SIMULATION}

Examples of accidental drops, related to the transportation or other kinds of fuel handling are presented in Fig. 1. One of the most important (from the safety point of view) accident is the FA impacting a rigid target. Deformations of the FA parts are quite complex in this case and partly unpredictable which is a result of the dimensions variation (even within allowable production tolerance) or slight differences in materials properties. Apart from that multiplicity of the interactions between FA parts also affects the results. As an example one can concern contact of the fuel pellets with cladding as well as the fuel rods and guide tubes with the spacers. Total amount of the fuel rods and guide tubes is 331 . Amount of spacers can vary from 13 to 15 . A spacer is a grid made of 312 individual cells connected via spotwelds and enclosed in a hexagonal band. Each cell is 20-30 mm high, has a hexagonal cross-section and is made of hollow tube with $0.3 \mathrm{~mm}$ wall thickness. The cell has also 3 dimples to keep a fuel rod equidistant from the cell walls. The geometry of a spacer and FA are shown in Fig. 1. 

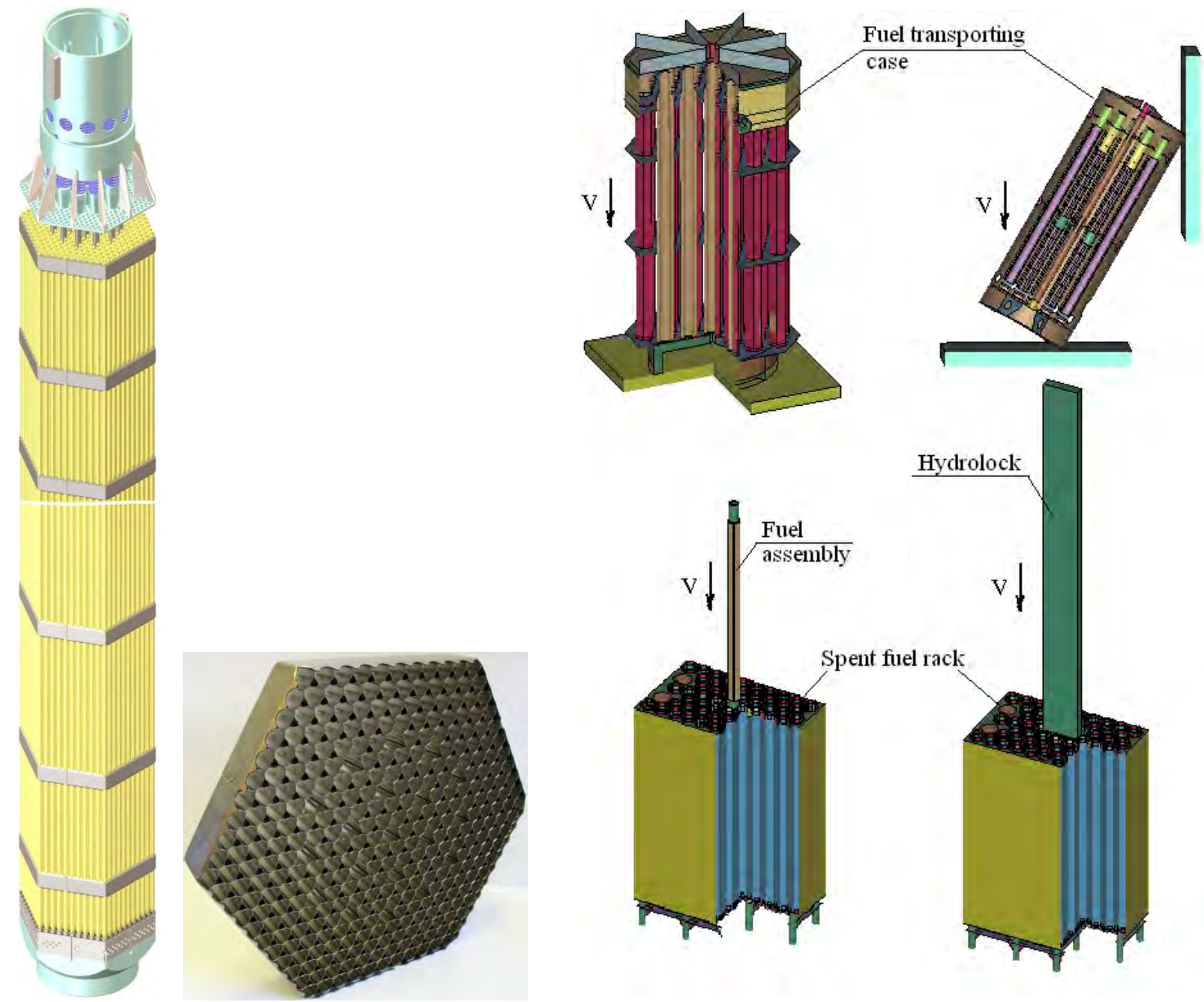

Fig. 1. FA geometry and examples of accidents

Therefore each fuel rod and guide tube makes 39 contact pairs with spacer cells. Total amount of contact pairs is $1,3 \cdot 10^{4}$. There are about 350 fuel pellets in a fuel rod. Each pellet contacts with cladding and neighboring pellets which results in approximately 700 contact pairs per fuel rod. Thus, minimal amount of contact pairs to be considered per FA in any situation is $700 \cdot 312+1,3 \cdot 104=2,3 \cdot 10^{5}$. Apart from that, results are greatly affected by material properties deviations. Realistic simulation of desired dynamic processes cannot be realized using so called guaranteed (worst possible) material properties as it would not be conservative.

Hence development of a full-scale FA model has to be based on thorough investigations of the FA individual parts behaviour. It can be done by making experiments with relatively simple models which represent a single process without its interference with the other ones. Moreover reliable dynamic material properties have to be established

To do this a series of experiments was conducted. Four models were developed for experimental investigations: two relatively simple models; a shortened model of a real FA, with two spacers and a weight on the top to simulate mass of missing fuel pellets and a real fuel assembly including lead imitators of fuel pellets. The latter is not discussed in this paper. Description of other models and the experimental facility are given in the following sections.

\section{EXPERIMENTAL SETUP}

The drop-test facility is a 15 meters high steel frame. It is shown schematically in Fig. 2. Maximal drop height is $13 \mathrm{~m}$. Weights were attached to the top of all models to increase their kinetic energy. On the side weights were attached to the guide rails to provide full contact of the models with the rigid target. 
The target is made of a $30 \mathrm{~mm}$ thick steel plate casted in a concrete block of 18 tons weight. The block is put on springs to suppress its interaction with a bed of surrounding building.

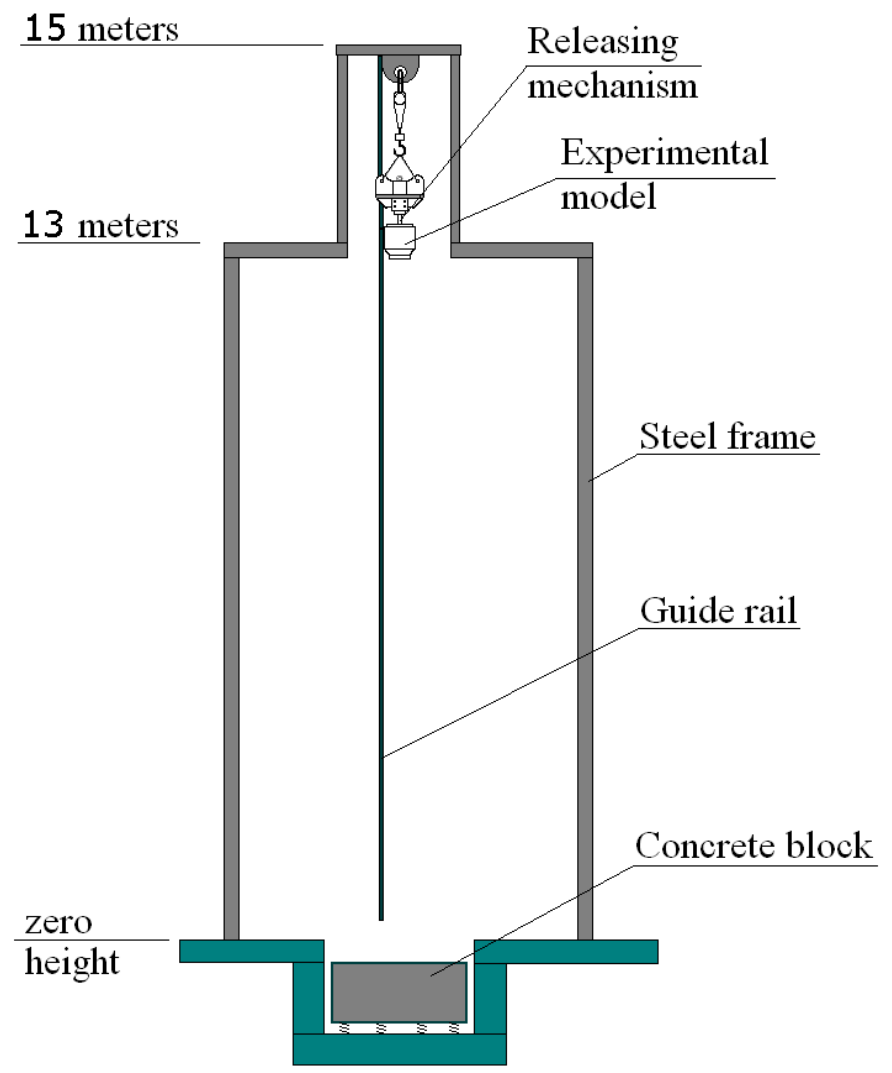

Fig. 2. Schematics of the experimental facility

\section{DESCRIPTION OF THE EXPERIMENTAL MODELS AND MEASUREMENTS PROCEDURE}

The target is made of a $30 \mathrm{~mm}$ thick steel plate casted in a concrete block of 18 tons weight. The block is put on springs to suppress its interaction with a basement of surrounding building.

Usage of small and simple experimental models allows their multiple production and more detailed investigation. First of all increased number of experiments provide better statistics for each of the problems. Moreover subsequent experiments can be corrected (drop height, weight mass etc.) using the previous results. This is particularly important as numerical simulation of dynamic processes during investigated impact loading can be problematic. Finite-elements models used for numerical simulation of the drop tests are shown in Fig. 3.

The first model is a simplified FA tail and is used and investigate large plastic deformations (or damage) under high speed impact loading. These data are necessary for the verification of FE models and approaches to simulate such kind of interactions.

The second model used to simulate a drop of the nuclear fuel transportation container loaded with a FA. This is used to verify approaches to numerical simulation of accidental drops which can occur during fuel transportation (within a container) with LS-Dyna software. The principal outcomes of the experiment are the plastic deformations and damages of the FA and container stainless steel models as well as the data concerning fuel and container accelerations during the impact with a rigid target.

The third model is the most complicated one. It is a lower part of a real FA. Previous vertical drop tests have shown that the lower part of FA undergoes large plastic deformations whilst the upper part remains almost intact. Therefore such model is sufficient to verify the finite-elements model which can be afterwards improved to be used for a full-scale FA drop test simulation. To simulate a missing mass of the FA's upper part a weight is attached on the top of the model. 
One of the experiment goals is to verify a finite-elements model developed with a reasonable minimum of simplifications. The major simplification is usage of shell elements to simulate fuel rods and spacer cells. A special attention was paid to the interaction of the edges with a perforated lower plate as the experiments have shown that their interpenetration and thus material damage takes place. The drop height was 2 meters.

Apart from the model itself Fig. 3 shows where strain gauges are placed: cylindrical part of a FA tail, fuel rods and gauges for measurements of transversal and longitudal strains of the edges welded to a tail (cannot be seen).

Weight deceleration after impact is one of the principal integral experimentally measured parameters of the drop test. It determines dynamics of the model's kinetic energy dissipation via plastic deformations of its components. Reliable prediction of deceleration is impossible without knowing real material properties and without proper description of the whole model. Deceleration of the weights was measured by accelerometers attached to them.

The investigated models were equipped with strain gauges to compare time dependent material strains with the numerical simulation results. Residual strains were also measured and compared with the numerical solution. The impact process was captured via high speed camera (1000 frames per second).
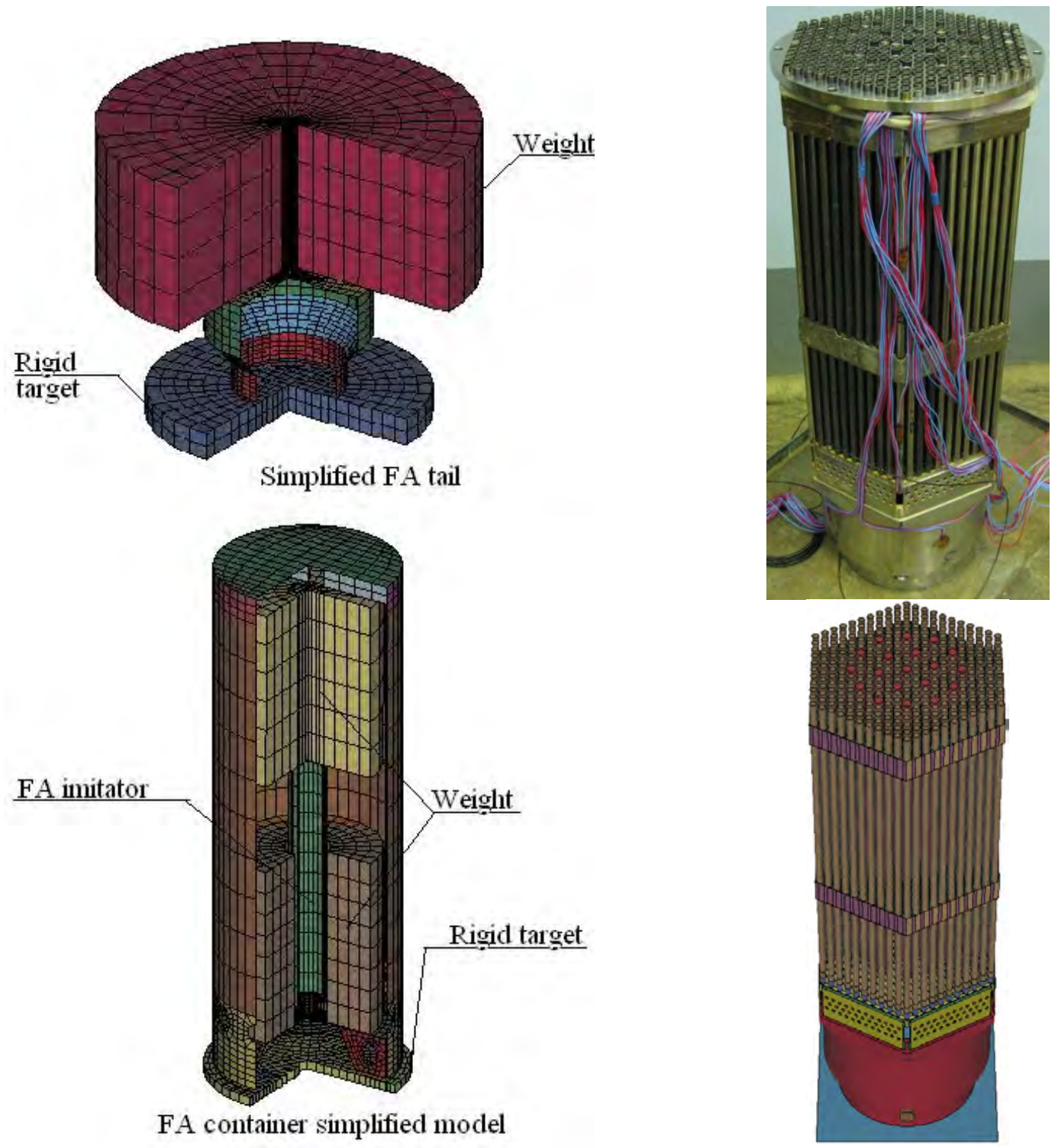

Fig. 3. Experimental models 


\section{EXPERIMENTAL AND NUMERICAL SIMULATION RESULTS}

Investigations of the simple models were conducted for the code verification purposes and for material properties establishment. All of the simulated results have shown a very good coincidence with the experimental data. The discussion of the large scale model experimental results and their comparison with the numerical simulation is given below.

Acceleration of the weight is shown in Fig. 4. A good agreement is obtained. Curve provided by the analysis is more smooth, as it's rather integral then a point characteristic. Otherwise moments of peak accelerations and the process duration are very well predicted by the analysis. The first acceleration maximum at $1.02 \mathrm{~ms}$ is a result of the edges and bottom grid interpenetration. At $2.17 \mathrm{~ms}$ tail starts to deform plastically which causes a second peak and slows the weight down to zero velocity.

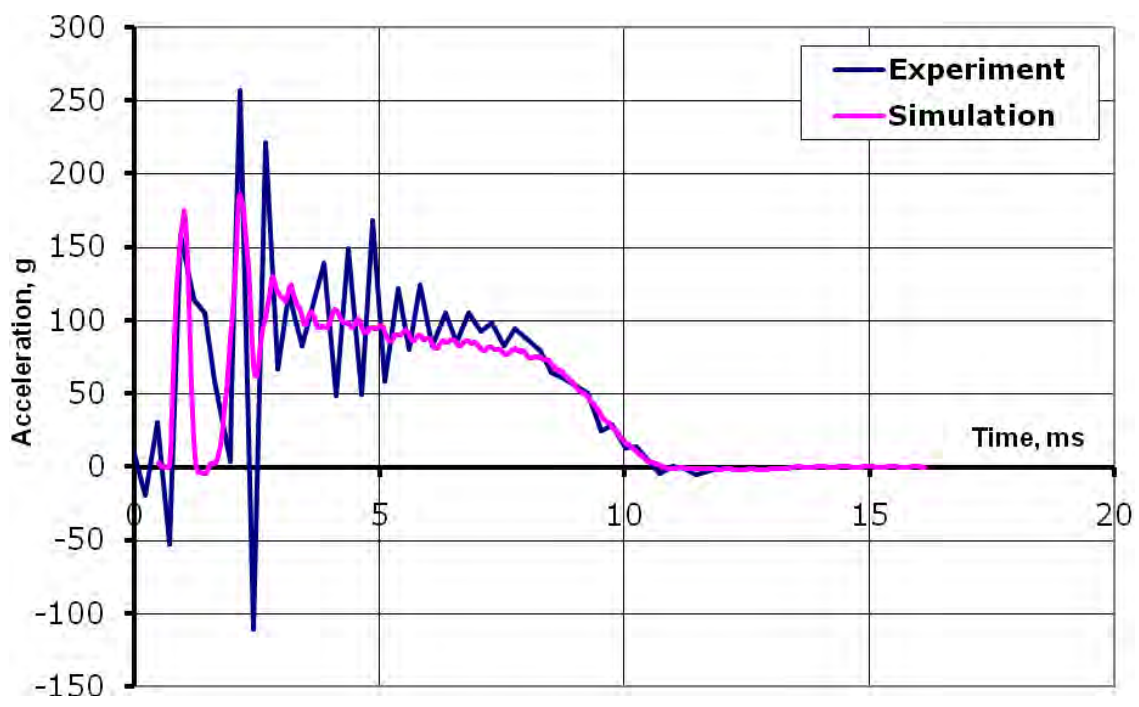

Fig. 4. Weight acceleration

Support edge was equipped with a strain gauge. Fig. 5 shows measured deformations and results of numerical simulation for the elements highlighted in Fig. 5a. good agreement is obtained both for the strain time history and for residual strain.

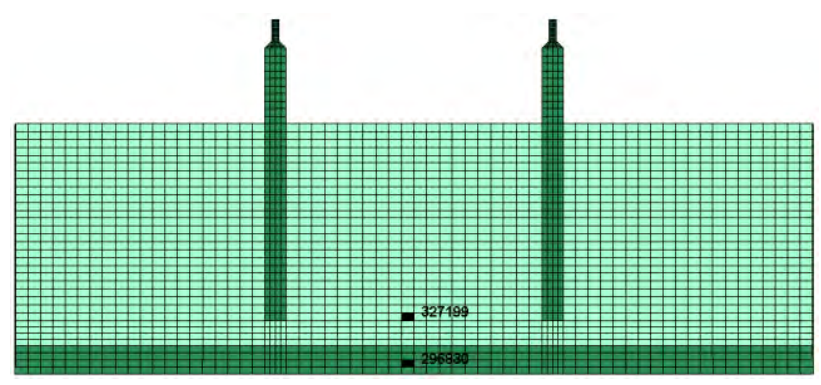

a

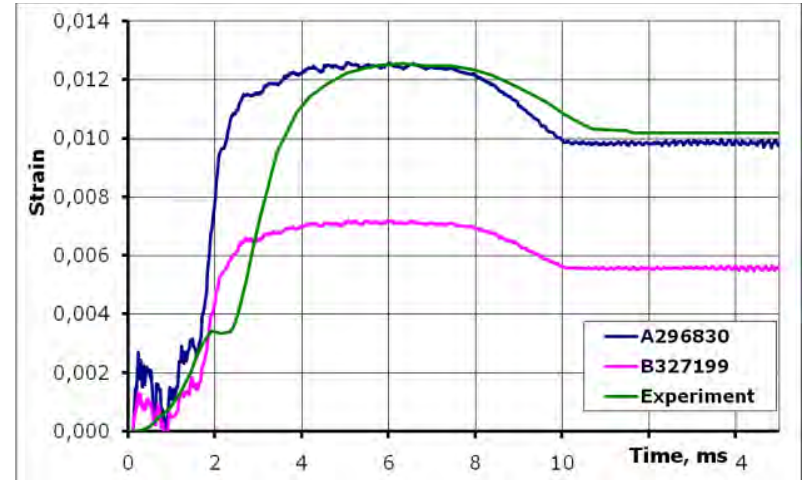

$\mathrm{b}$

Fig. 5. Time history of the lower edge strain

Comparison of cylindrical part axial deformations is shown in Fig. 6. Strain gauges 55-58 are equally spaced on the tail's outer surface. It is clearly seen that experimental data differs from point to point. This can results from deformation's circumferential nonuniformity. Moreover the analysis has shown that tail's axial strains depend strongly on height, which can also lead to the scatter of results from different gauges. In average quite good 
agreement is obtained for the deformations time history. The discrepancies can be explained be the imperfectness of the experimental model, slight differences in material properties, discretization of the FE model or by difference between measuring technique and analysis postprocessing.

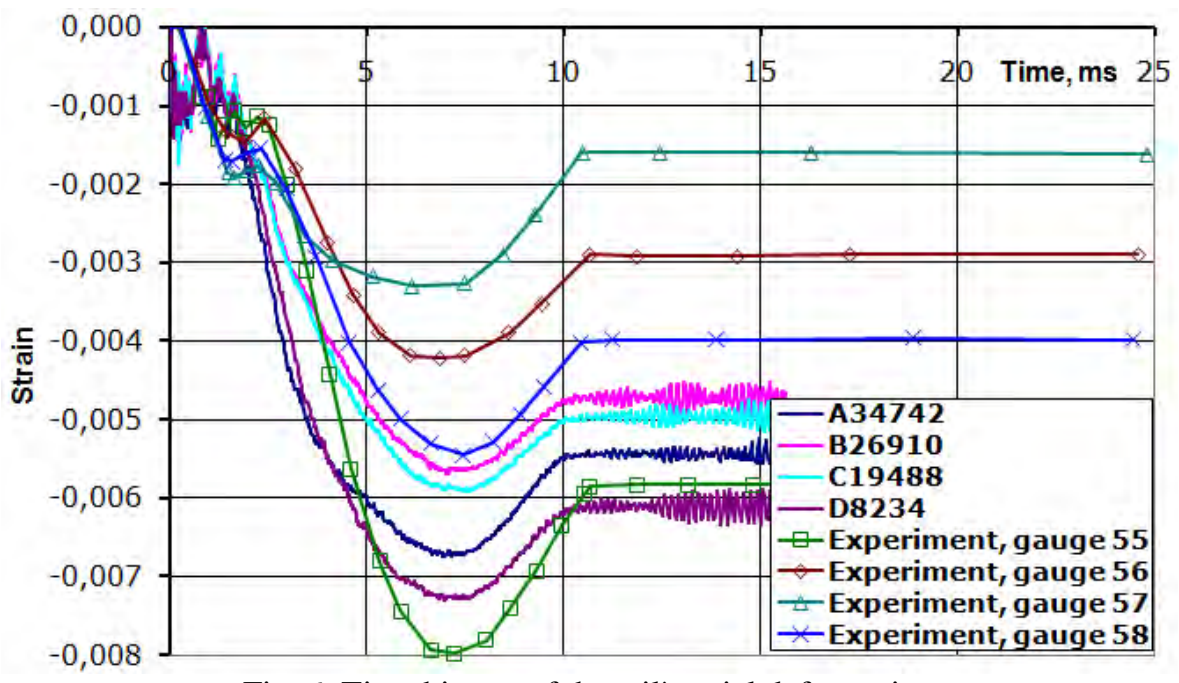

Fig. 6. Time history of the tail's axial deformation

Deformations of the fuel rods cladding were measured by strain gauges placed on the corner rods in between the spacers. Numbering and position of gauges is given in Table 1 and in Fig. 7. Numbering of rods and sides is done clockwise (when being viewed from above) starting from the fixation pin. Comparison of the solution with the experimental date is shown in Fig. 7 and Fig. 8. In general good agreement is obtained. Peak strains at $1 \mathrm{~ms}$ are very well predicted. difference in residual stresses can arise from either insufficient bottom grid FE refinement or by using shell elements to model the fuel rods.

Table 1: Positioning of strain gauges

\begin{tabular}{|c|l|}
\hline Strain gauge number & Position \\
\hline 59 & fuel rod 1 , side 3 , in between spacers 1 and 2 , axial direction \\
\hline 60 & fuel rod 7, side 4 , in between spacers 1 and 2 , axial direction \\
\hline 63 & fuel rod 1, side 1 , in between spacers 1 and 2 , axial direction \\
\hline 64 & fuel rod 1, side 1 , in between spacers 2 and 3 , axial direction \\
\hline
\end{tabular}

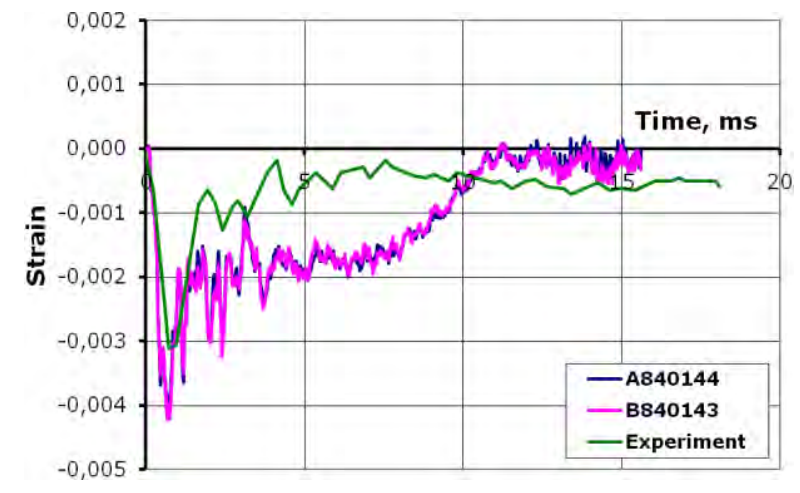

a

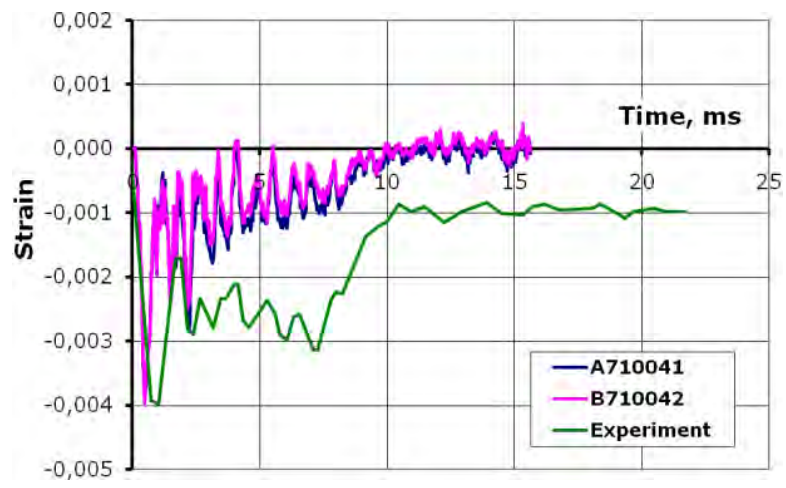

$\mathrm{b}$

Fig. 7. Fuel rod shells axial deformations for gauges: $a-59$ and $b-60$

As it was mentioned edges penetrated bottom grid by destruction of thin walls between the holes. As a result gap between the tail and bottom grid decreased by $10,5 \mathrm{~mm}$. Numerically simulated value is $11.3 \mathrm{~mm}$ which is very close to the experimental one. Deformed FA is shown in Fig. 9. 


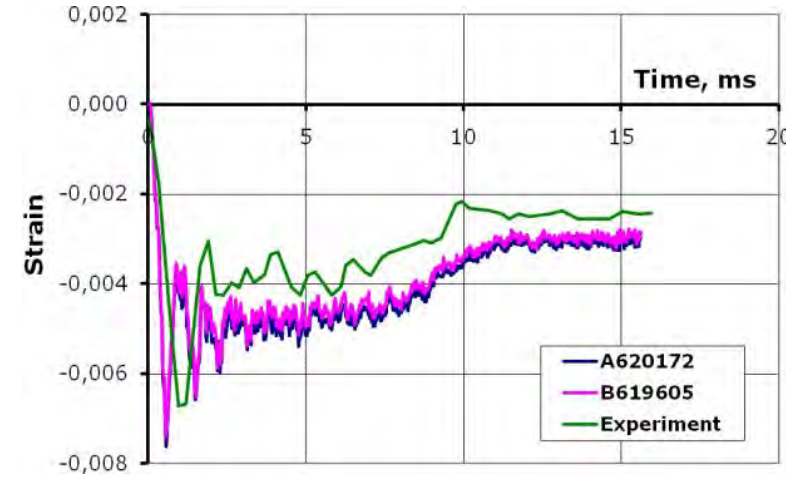

a

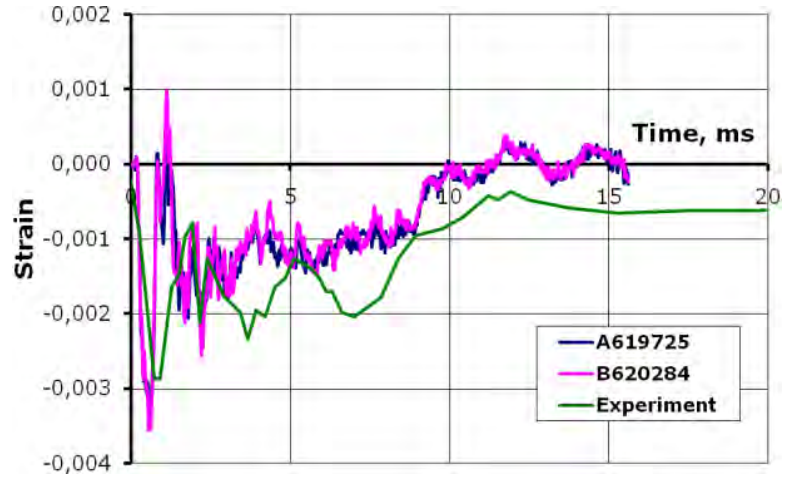

b

Fig. 8. Fuel rod shells axial deformations for gauges: $a-63$ and $b-64$
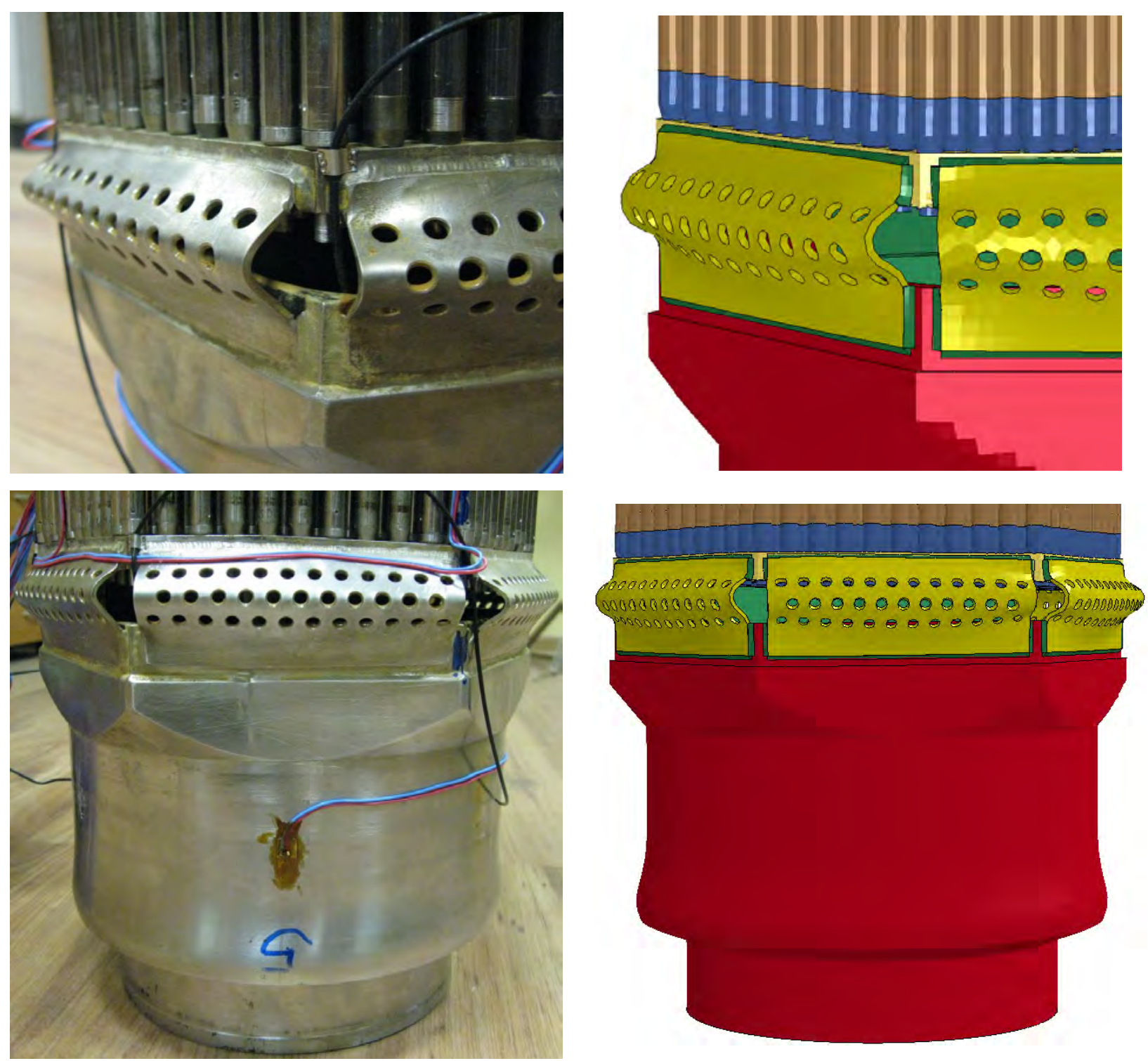

a

Fig. 9. Deformed FA: a - experiment, b - simulation 


\section{CONCLUSION}

The present research was devoted to the application of LS-Dyna software for analysis related to the nuclear safety during nuclear fuel transportation accidents. The principal problem is to define deformed states of the fuel assembly during and after the accident. Series of experiments were conducted and numerically simulated. It has been shown that reliable solution can be obtained for a large scale model of a real FA. This means that newly developed nuclear fuel assemblies can be numerically investigated without conducting expensive and complicated experiments.

\section{REFERENCES}

[1] LS-DYNA Keyword User's Manual. Version 971. LSTC, 2007.

[2] LS-DYNA Theory Manual. Version 971. LSTC, 2006. 